\title{
ChemComm
}

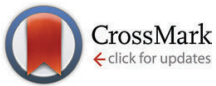

Cite this: Chem. Commun., 2015, 51,6384

Received 16th January 2015 Accepted 26th February 2015

DOI: $10.1039 / \mathrm{c} 5 \mathrm{cc} 00454 \mathrm{c}$

www.rsc.org/chemcomm

\section{Linifanib - a multi-targeted receptor tyrosine kinase inhibitor and a low molecular weight gelator $\dagger$}

\author{
Maria Marlow, * Mohammed Al-Ameedee, Thomas Smith, Simon Wheeler and \\ Michael J. Stocks
}

\begin{abstract}
In this study we demonstrate that linifanib, a multi-targeted receptor tyrosine kinase inhibitor, with a key urea containing pharmacophore, self-assembles into a hydrogel in the presence of low amounts of solvent. We demonstrate the role of the urea functional group and that of fluorine substitution on the adjacent aromatic ring in promoting self-assembly. We have also shown that linifanib has superior mechanical strength to two structurally related analogues and hence increased potential for localisation at an injection site for drug delivery applications.
\end{abstract}

In recent years, there has been significant interest focused on the use of low molecular weight gelators (LMWGs) for medical applications. ${ }^{1-3}$ Scientists working in this field are evaluating LMWGs, which form hydrogels, for drug delivery and tissue engineering applications ${ }^{4,5}$ and indeed there are FDA approved molecular hydrogels for example, Lanreotide. ${ }^{6}$ To create these hydrogels, LMWGs self-assemble into three dimensional, fibrillar networks that have large quantities of water associated with them. ${ }^{2}$ In the presence of low stress, these systems exhibit solidlike behaviour even though they have a low solid content, typically less than $2 \%(\mathrm{w} / \mathrm{w})$. The properties of large amounts of associated water and low solid content will confer biocompatibility, ${ }^{4}$ making these systems attractive for drug delivery. One specific application of LMWGs is cancer therapeutics, where they have been used to localize and give controlled release of chemotherapeutics upon intra-tumoural injection, as exemplified by Wang et al. ${ }^{7,8}$ Our studies have extended beyond chemotherapeutics to that of molecularly targeted drugs, such as vascular endothelial growth factor (VEGF) inhibitors. ${ }^{9}$ We have selected one such compound linifanib (1, Fig. 1), a potent dual vascular endothelial growth factor (VEGF) and platelet-derived growth factor (PDGF) tyrosine kinase inhibitor, and was a drug candidate for the treatment of advanced or metastatic non-small cell lung cancer, renal cancer

School of Pharmacy, University of Nottingham, University Park, Nottingham, NG7 2RD, UK. E-mail: maria.marlow@nottingham.ac.uk

$\dagger$ Electronic supplementary information (ESI) available: Experimental procedures, NMR spectra, and rheology data. See DOI: 10.1039/c5cc00454c and pancreatic cancer. ${ }^{10} \mathrm{We}$ chose, in part, to investigate the gelation properties of linifanib as pancreatic cancer is poorly treated by systemic therapies due to poor drug penetration of the stromal barrier ${ }^{11}$ and we considered that a LMWG hydrogel of such a therapy, injected intra-tumourally, could be considered as a novel approach to increase such poor drug penetration. This linifanib hydrogel should confer biocompatibility to the intra-tumoural depot due to the large amounts of associated water. ${ }^{4}$ We have also chosen linifanib as the molecule has nanomolar potency and hence will only require dissolution of nanomolar concentrations to achieve efficacy. ${ }^{12}$ Another consideration in the selection of this drug was the class of LMWGs that this drug also represents. LMWGs with a urea moiety, which is a key part of the pharmacophore of linifanib, have not been extensively studied for drug delivery. ${ }^{13}$ Indeed, the focus has been on using LMWGs derived from peptides, nucleosides or carbohydrates. ${ }^{14}$ The urea functional group is both a hydrogen bond donor and acceptor group, known to create the one dimensional order required for supramolecular networks. ${ }^{13,15}$ Hydrogen bonds are formed between the oxygen atom of the carbonyl group and hydrogen atoms of the two $\mathrm{NH}$ groups resulting in directional assembly. Also hydrogen bonding contributes to drug-receptor interactions and hence the urea functional group, with its extensive hydrogen bonding potential has been incorporated within many

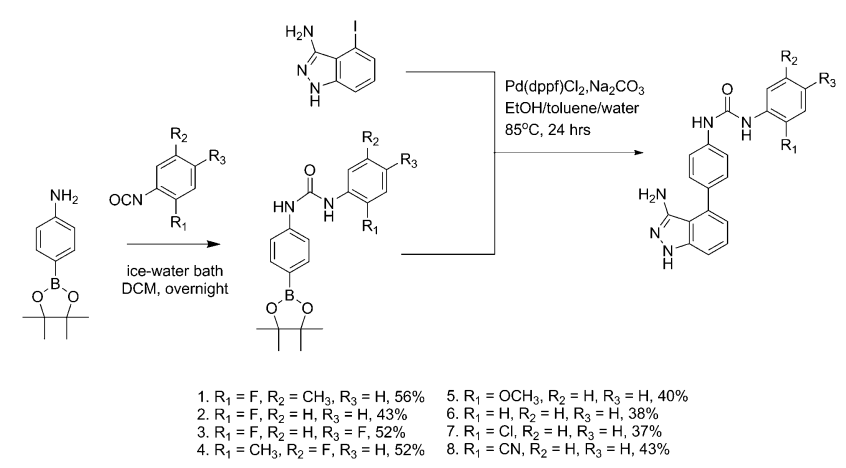

Fig. 1 Linifanib (1) and analogues (2-8) by modification of substituents on the terminal aromatic ring adjacent to the urea. 

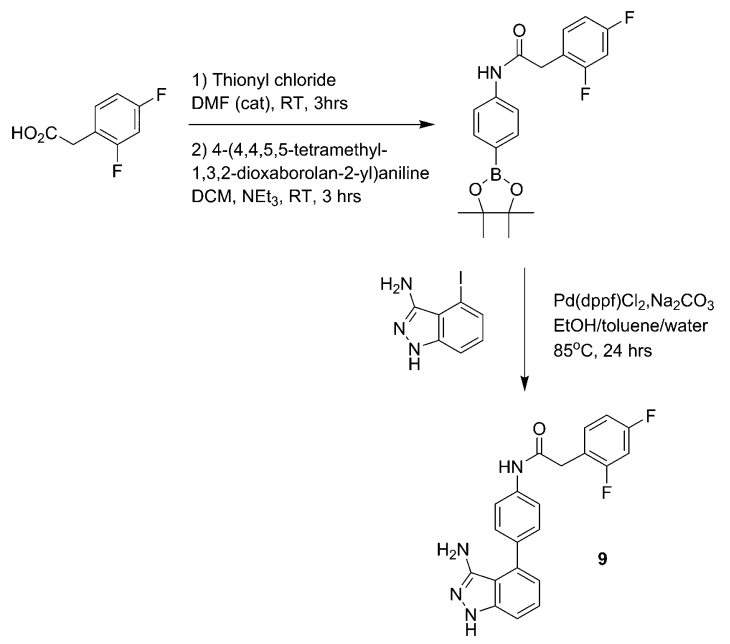

Fig. 2 Analogue (9) by replacement of the urea functional group by an amide.

drug pharmacophores to give both potency and specificity. Indeed, we speculate that there may be many drug molecules containing the urea functional group that are LMWGs.

We also selected linfinab due to the presence of the aromatic rings flanking the urea functional group, leading to $\pi-\pi$ stacking interactions which in turn have the potential to promote selfassembly. ${ }^{16}$ Based on our hypothesis of both the urea functional group and the aromatic rings promoting gelation in linifanib, we synthesized seven close analogues by modification of the substituents on the terminal aromatic ring adjacent to the urea (2-8) (Fig. 1) and an additional analogue containing an amide group instead of the urea group (9) (Fig. 2). The amide was chosen since it also can provide complimentary hydrogen bonding interactions required to promote self-assembly. ${ }^{2}$ Linifanib (1) and all eight analogues were screened for gelation using "table top rheology" i.e. stable to vial inversion. ${ }^{17}$ Gels were formed by dissolving the compounds in DMSO, followed by adding water at $60{ }^{\circ} \mathrm{C}$. The results of the vial inversion screen are shown in Table 1 where the final gelator concentration is $0.5 \%(\mathrm{w} / \mathrm{v})$, which is common for a $\mathrm{LMWG}{ }^{2}$ in dimethyl sulfoxide (DMSO)-water mixtures from $5 \%(\mathrm{v} / \mathrm{v})$ to $25 \%(\mathrm{v} / \mathrm{v})$. The solvent mixture was chosen as linifanib is soluble in DMSO but insoluble in water at the required concentration; there is significant literature precedent for using this anti-solvent approach; ${ }^{18,19}$ and increased concentrations of DMSO are potentially advantageous in cancer where DMSO has been shown to reduce the tumourigenicity of human pancreatic cell lines. ${ }^{20}$ Using water as the anti-solvent we were able to reproducibly induce gelation for linifanib (1) and the synthesised analogues that contained a fluorine atom substituted on the aromatic ring and the urea functional group in the structure $(2,3$ and 4$)$. The gels that formed were stable to vial

Table 1 Vial inversion screen of $0.5 \%(\mathrm{w} / \mathrm{v})$ linifanib and linifanib analogues in DMSO-water mixtures, where a gel (G), or a precipitate (P) was formed

\begin{tabular}{llllllllll}
\hline Compound v/v DMSO-water & 1 & 2 & 3 & 4 & 5 & 6 & 7 & 8 & 9 \\
\hline 5 & G & G & G & G & P & P & P & P & P \\
10 & G & G & G & G & P & P & P & P & P \\
15 & G & P & G & G & P & P & P & P & P \\
20 & P & P & G & P & P & P & P & P & P \\
25 & P & P & G & P & P & P & P & P & P
\end{tabular}

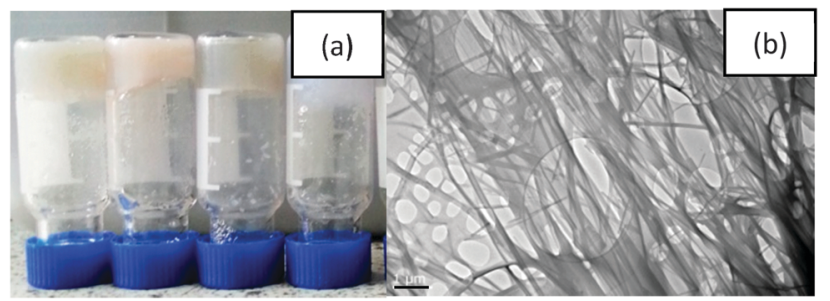

Fig. 3 (a) Vial inversion screen of linifanib and its fluorinated analogues (left to right: linifanib (1) and linifanib analogues 2, $\mathbf{3}$ and $\mathbf{4}$ ) (b) transmission electron micrograph (TEM): $0.5 \%(\mathrm{w} / \mathrm{v})$ linifanib in $5 \%(\mathrm{v} / \mathrm{v})$ DMSO-water; scale bar $=1 \mu \mathrm{m}$.

inversion (Fig. 3). Moreover, the role of the urea functional group to promote gelation was demonstrated by comparison of the gelation behavior of $\mathbf{3}$ and $\mathbf{9}$. This structural change from urea (gel) to amide (precipitate) in the vial inversion screen demonstrated the important role that the urea functional group plays in the gelation of linifanib and its structural analogues. However, the 2-methoxy analogue (5) and the un-substituted terminal aromatic ring analogue (6), formed precipitates under all the conditions and likewise both the 2-chloro analogue (7) and the nitrile analogue (8) failed to gel, even though they both had electron-withdrawing substituents on the terminal aromatic ring. These observations are not surprising given the previously reported data of Nilsson et al., ${ }^{16}$ who showed that minor substitutions on the aromatic ring of Fmoc-protected aromatic amino acids influenced gelation. They demonstrated the effect was a result of both steric and electronic changes in $\pi-\pi$ stacking interactions.

Specifically, they describe how a fluorine atom substituted on a toluene ring (also present in the terminal aromatic ring of linifanib) can create a more electron deficient aromatic ring as compared to substitution with other halogens and also fluorine has a beneficial smaller molecular volume. Also described in the Nilsson paper is that the halogen identity and position of substitution on the ring influences the gel strength. We also found in our studies that linifanib (1), with an ortho substituted fluorine, gave stronger gels as compared to its fluorine substituted analogues (2, 3 (di-fluoro substituted) and $\mathbf{4}$ (meta substituted fluorine)) respectively in the vial inversion screen and confirmed by rheology for analogue 3 (see S3, $\mathrm{ESI} \dagger$ ). Linifanib was selected as the focus for further physical characterization dues it greater mechanical strength as this is an important attribute for a drug delivery applications where mechanical strength can influence localization at an injection site. ${ }^{21,22}$

Firstly, further vial inversion screening for gelation was carried out with a series of binary mixtures of DMSO and water (Table 2). These results indicate that DMSO : water ratio had a significant effect on gelation which has also been observed for other LMWGs. ${ }^{18,23}$ The linifanib gels that were most stable to vial inversion were obtained with $5 \%(\mathrm{v} / \mathrm{v})$ DMSO-water. Interestingly, there seems to be an optimum ratio of linifanib to DMSO in that too much DMSO disrupts the hydrogen bonding interactions. Hence, at a low solvent ratio $(5 \%(\mathrm{v} / \mathrm{v}))$, we confirmed the fibrillar structure of the linifanib gels by the transmission electron microscopy (TEM). $0.5 \%(\mathrm{w} / \mathrm{v})$ linifanib, demonstrated a branched pattern of fibres 100 to $300 \mathrm{~nm}$ in width and 4.5 to 9 microns in length (Fig. 3). 
Table 2 Vial inversion screen of linifanib in binary mixtures of DMSO and water where gel $(G)$, solution $(S)$ and precipitate $(P)$

\begin{tabular}{llll}
\hline & \multicolumn{2}{l}{$\%(\mathrm{w} / \mathrm{v})$ linifanib } & \\
\cline { 2 - 4 }$\%(\mathrm{v} / \mathrm{v}) \mathrm{DMSO}$ & 0.125 & 0.25 & 0.5 \\
\hline 5 & $\mathrm{G}$ & $\mathrm{G}$ & $\mathrm{G}$ \\
10 & $\mathrm{P}$ & $\mathrm{G}$ & $\mathrm{G}$ \\
15 & $\mathrm{P}$ & $\mathrm{P}$ & $\mathrm{G}$ \\
20 & $\mathrm{~S}$ & $\mathrm{P}$ & $\mathrm{P}$ \\
25 & $\mathrm{~S}$ & $\mathrm{~S}$ & $\mathrm{P}$ \\
30 & $\mathrm{~S}$ & $\mathrm{~S}$ & $\mathrm{~S}$ \\
40 & $\mathrm{~S}$ & $\mathrm{~S}$ & $\mathrm{~S}$ \\
50 & $\mathrm{~S}$ & $\mathrm{~S}$ & $\mathrm{~S}$ \\
\hline
\end{tabular}

Having confirmed the characteristic LMWG fibrillar network, we then undertook oscillatory rheology experiments with linifanib concentrations from 0.0625 to $1.25 \%(\mathrm{w} / \mathrm{v})$ in $5 \%(\mathrm{v} / \mathrm{v})$ DMSOwater. The measurements were performed at strains of $0.01 \%$ to $100 \%$ strain at $10 \mathrm{rad} \mathrm{s}^{-1}$ and the storage modulus $\left(G^{\prime}\right)$ and loss modulus $\left(G^{\prime \prime}\right)$ are reported from within the linear viscoelastic region (Fig. 4). These experiments provided key evidence of gelation of linifanib in DMSO-water as demonstrated by the $G^{\prime}$ exceeding $G^{\prime \prime}$ by at least an order of magnitude proving the viscoelastic nature of the gel. ${ }^{24}$ Additionally, a sustained linear viscoelastic region was found between $0.01-1 \%$ (see S3, ESI $\dagger$ ). Our $G^{\prime}$ data $\left(1.5 \times 10^{4} \mathrm{~Pa}\right)$ is lower than the gels $\left(10^{6} \mathrm{~Pa}\right)$ as reported Kleinsmann ${ }^{25}$ and $\mathrm{Yu}^{24}$ but greater than the $G^{\prime} 100 \mathrm{~Pa}$ of gels as reported by Ren $^{26}$ for a Gemcitabine LMWG. An increase in the moduli with increasing concentration of linifanib was also observed which has likewise been shown for other LMWGs, for example the simple chiral bisureas of Rodriguez-Llansola et $a .^{27}$ Additionally, in a similar manner to Adams et al., ${ }^{28}$ we evaluated the power law relationship between $G^{\prime}$ and gelator concentration i.e. $G^{\prime} \propto C^{x}$ (see S3, ESI $\dagger$, Fig. 3). We found that $x$ was 1.86 indicative of a semi-entangled network.

In summary, we have demonstrated that the multi-targeted receptor tyrosine kinase inhibitor, linifanib, with a urea containing pharmacophore, is a LMWG and forms a hydrogel in low solvent ratios of DMSO-water. Representative gels were studied using rheology and TEM and their fibrillar structure confirmed.

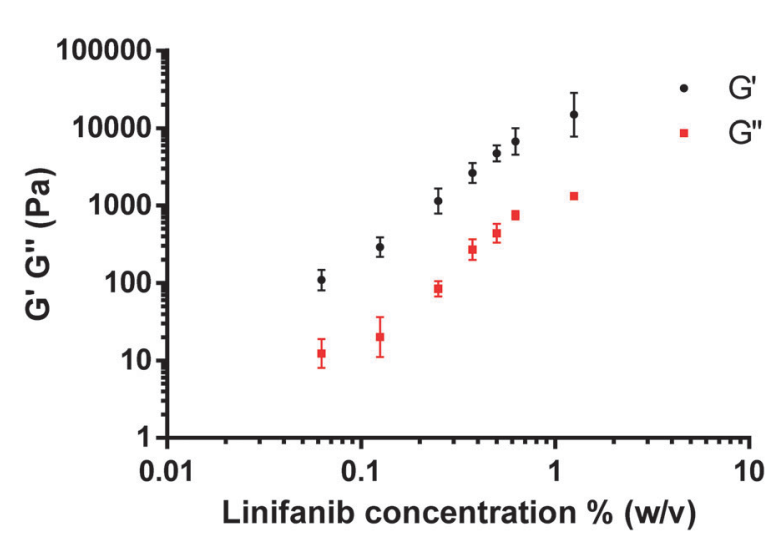

Fig. 4 Storage modulus $\left(G^{\prime}\right)$ (black circle) and loss modulus $\left(G^{\prime \prime}\right)$ (red square) versus linifanib concentration $(\%(\mathrm{w} / \mathrm{v}))$ in $0.5 \%(\mathrm{v} / \mathrm{v}) \mathrm{DMSO}-$ water. All measurements were carried out in triplicate and the mean calculated. Error bars represent standard deviation.
Through the synthesis of structurally related analogues we have also shown the importance of both the urea functional group and position of the essential fluorine substitution on the terminal aromatic ring in promoting gelation. We also shown that linifanib has superior mechanical strength its structurally related analogues and hence increased potential for localisation at an injection site for drug delivery applications.

The authors declare no competing financial interests.

Support from Mike Fay at the Nottingham Nanotechnology and Nanoscience Centre (NNNC) for the TEM Imaging. We also thank the Government of Iraq for financial support (to Mohammed Al-Ameedee) and the University of Mustansiriya, Baghdad, Iraq and The Wellcome Trust for funding a summer student bursary to Thomas Smith.

\section{Notes and references}

$1 \mathrm{~J}$. W. Steed, Supramolecular gel chemistry: developments over the last decade, Chem. Commun., 2011, 47(5), 1379-1383.

2 L. A. Estroff and A. D. Hamilton, Water gelation by small organic molecules, Chem. Rev., 2004, 104(3), 1201-1217.

3 R. G. Weiss, The Past, Present, and Future of Molecular Gels. What Is the Status of the Field, and Where Is It Going?, J. Am. Chem. Soc., 2014, 136(21), 7519-7530.

4 K. J. Skilling, F. Citossi, T. D. Bradshaw, M. Ashford, B. Kellam and M. Marlow, Insights into low molecular mass organic gelators: a focus on drug delivery and tissue engineering applications, Soft Matter, 2014, 10(2), 237-256.

5 R. Tian, J. Chen and R. Niu, The development of low-molecular weight hydrogels for applications in cancer therapy, Nanoscale, 2014, 6, 3474-3482.

6 E. Prommer, Established and potential therapeutic applications of octreotide in palliative care, Support Care Cancer, 2008, 16(10), 1117-1123.

7 H. M. Wang, J. Wei, C. B. Yang, H. Y. Zhao, D. X. Li, Z. N. Yin and Z. M. Yang, The inhibition of tumor growth and metastasis by selfassembled nanofibers of taxol, Biomaterials, 2012, 33(24), 5848-5853.

8 H. M. Wang, L. N. Lv, G. Y. Xu, C. B. Yang, J. T. Sun and Z. M. Yang, Molecular hydrogelators consist of taxol and short peptides/amino acids, J. Mater. Chem., 2012, 22(33), 16933-16938.

9 H. Z. Zhong and J. P. Bowen, Recent Advances in Small Molecule Inhibitors of VEGFR and EGFR Signaling Pathways, Curr. Top. Med. Chem., 2011, 11(12), 1571-1590.

10 B. C. Goh, C. Wong, J. Qian, E. McKeegan, J. L. Ricker, D. M. Carlson and R. A. Soo, Extended linifanib therapy in patients with advanced solid tumors in a phase I trial, J. Clin. Oncol., 2010, 28(suppl 15), 3053.

$11 \mathrm{~A}$. H. I. Al Haddad and T. E. Adrian, Challenges and future directions in therapeutics for pancreatic ductal adenocarcinoma, Expert Opin. Invest. Drugs, 2014, 23(11), 1499-1515.

12 Linifanib, Drugs R\&D, 2010, 10(2), 111-122.

13 J. W. Steed, Anion-tuned supramolecular gels: a natural evolution from urea supramolecular chemistry, Chem. Soc. Rev., 2010, 39(10), 3686-3699.

14 B. Xu, Gels as Functional Nanomaterials for Biology and Medicine, Langmuir, 2009, 25(15), 8375-8377.

15 M. Yamanaka, Urea derivatives as low-molecular-weight gelators, J. Inclusion Phenom. Macrocyclic Chem., 2013, 77(1-4), 33-48.

16 D. M. Ryan, S. B. Anderson and B. L. Nilsson, The influence of sidechain halogenation on the self-assembly and hydrogelation of Fmoc-phenylalanine derivatives, Soft Matter, 2010, 6(14), 3220-3231.

17 S. P. Raghaven and B. H. Cipriano, Gel Formation:Phase Diagrams using Table Top Rheology and Calorimetry, in Molecular Gels. Materials with Self-Assembled Fibrillar Networks, ed. P. Terech and R. G. Weiss, Springer, Dordrecht, 2006, pp. 241-243.

18 J. Raeburn, G. Pont, L. Chen, Y. Cesbron, R. Levy and D. J. Adams, Fmoc-diphenylalanine hydrogels: understanding the variability in reported mechanical properties, Soft Matter, 2012, 8(4), 1168-1174.

19 M. Rodrigues, A. C. Calpena, D. B. Amabilino, M. L. GardunoRamirez and L. Perez-Garcica, Supramolecular gels based on a 
gemini imidazolium amphiphile as molecular material for drug delivery, J. Mater. Chem. B, 2014, 2(33), 5419-5429.

20 L. J. McIntyre and Y. S. Kim, Eur. J. Cancer Clin. Oncol., 1984, 20, 265-271.

21 Y. Loo, S. Zhang and C. A. E. Hauser, From short peptides to nanofibers to macromolecular assemblies in biomedicine, Biotechnol. Adv., 2012, 30(3), 593-603.

22 S. Kempe and K. Mader, In situ forming implants - an attractive formulation principle for parenteral depot formulations, $J$. Controlled Release, 2012, 161(2), 668-679.

23 N. A. Dudukovic and C. F. Zukoski, Evidence for equilibrium gels of valence-limited particles, Soft Matter, 2014, 10(39), 7849-7856.

24 G. Yu, X. Yan, C. Han and F. Huang, Characterization of supramolecular gels, Chem. Soc. Rev., 2013, 42(16), 6697-6722.
25 A. J. Kleinsmann, N. M. Weckenmann and B. J. Nachtsheim, PhosphateTriggered Self-Assembly of $N$-[(uracil-5-yl)methyl]urea: A Minimalistic Urea-Derived Hydrogelator, Chem. - Eur. J., 2014, 20(31), 9753-9761.

26 C. Ren, C. Xu, D. Li, H. Ren, J. Hao and Z. Yang, Gemcitabine induced supramolecular hydrogelations of aldehyde-containing short peptides, RSC Adv., 2014, 4(66), 34729-34732.

27 F. Rodriguez-Llansola, D. Hermida-Merino, B. Nieto-Ortega, F. J. Ramirez, J. T. L. Navarrete, J. Casado, I. W. Hamley, B. Escuder, W. Hayes and J. F. Miravet, Self-Assembly Studies of a Chiral Bisurea-Based Superhydrogelator, Chem. - Eur. J., 2012, 18(46), 14725-14731.

28 J. Raeburn, C. Mendoza-Cuenca, B. N. Cattoz, M. A. Little, A. E. Terry, A. Zamith Cardoso, P. C. Griffiths and D. J. Adams, The effect of solvent choice on the gelation and final hydrogel properties of Fmocdiphenylalanine, Soft Matter, 2015, 11, 927-935. 